\title{
Progression of Amyloid Pathology to Alzheimer's Disease Pathology in an Amyloid Precursor Protein Transgenic Mouse Model by Removal of Nitric Oxide Synthase 2
}

\author{
Donna M. Wilcock, ${ }^{1}$ Matthew R. Lewis, ${ }^{1}$ William E. Van Nostrand, ${ }^{2}$ Judianne Davis, ${ }^{2}$ Mary Lou Previti, ${ }^{2}$ \\ Nastaran Gharkholonarehe, ${ }^{1}$ Michael P. Vitek, ${ }^{1}$ and Carol A. Colton ${ }^{1}$ \\ ${ }^{1}$ Division of Neurology, Duke University Medical Center, Durham, North Carolina 27710, and ${ }^{2}$ Department of Medicine, Stony Brook University, Stony \\ Brook, New York 11794
}

\begin{abstract}
Alzheimer's disease (AD) is characterized by three primary pathologies in the brain: amyloid plaques, neurofibrillary tangles, and neuron loss. Mouse models have been useful for studying components of AD but are limited in their ability to fully recapitulate all pathologies. We crossed the APPSwDI transgenic mouse, which develops amyloid $\beta(\mathrm{A} \beta)$-protein deposits only, with a nitric oxide synthase 2 (NOS2) knock-out mouse, which develops no AD-like pathology. APPSwDI/NOS2 ${ }^{-1-}$ mice displayed impaired spatial memory compared with the APPSwDI mice, yet they have unaltered levels of A $\beta$. APPSwDI mice do not show tau pathology, whereas APPSwDI/NOS2 ${ }^{-1-}$ mice displayed extensive tau pathology associated with regions of dense microvascular amyloid deposition. Also, APPSwDI mice do not have any neuron loss, whereas the APPSwDI/NOS2 ${ }^{-1-}$ mice have significant neuron loss in the hippocampus and subiculum. Neuropeptide $\mathrm{Y}$ neurons have been shown to be particularly vulnerable in AD. These neurons appear to be particularly vulnerable in the APPSwDI/ NOS2 ${ }^{-I-}$ mice as we observe a dramatic reduction in the number of NPY neurons in the hippocampus and subiculum. These data show that removal of NOS2 from an APP transgenic mouse results in development of a much greater spectrum of AD-like pathology and behavioral impairments.
\end{abstract}

Key words: amyloid; tau; neurodegeneration; neuropeptide Y; cerebral amyloid angiopathy; Alzheimer's disease

\section{Introduction}

Three pathologies must be present in the brain for a definitive diagnosis of Alzheimer's disease $(\mathrm{AD})$ : amyloid plaques composed of aggregates of amyloid $\beta$ (A $\beta$ ) peptides; neurofibrillary tangles composed of hyperphosphorylated, aggregated tau; and neuron loss (Nagy et al., 1998). The amyloid hypothesis proposes that $\beta$-amyloid accumulation is toxic to the brain resulting in hyperphosphorylation of tau, neuronal death, and cognitive deficits (Hardy and Selkoe, 2002). Transgenic mouse models expressing mutant human amyloid $\beta$ precursor protein (APP) successfully produce amyloid plaques and some cognitive decline but lack significant neuron loss and any tau pathology (Games et al., 1995; Hsiao et al., 1996; Hsiao, 1998; Schwab et al., 2004; Eriksen and Janus, 2007). Transgenic mouse models expressing human tau with mutations associated with frontotemporal dementia display tau pathology, but they fail to show amyloid deposition (Lewis et al., 2000; Santacruz et al., 2005). Transgenic

Received Nov. 14, 2007; revised Dec. 21, 2007; accepted Dec. 22, 2007.

This work was supported by National Institutes of Health Grants AG030942 (D.M.W.), NS55118 (W.E.V.N.), AG19780 (M.P.V.), and AG19740 (C.A.C.). Antibody reagents for the A $\beta$ ELISAs were generously provided by Lilly Research Laboratories. M.P.V. is a principal and founder of Cognosci.

The authors declare no competing financial interests.

Correspondence should be addressed to Carol A. Colton, Division of Neurology, Duke University Medical Center, Bryan Research Building, Box 2900, Research Drive, Durham, NC 27710. E-mail: glia01@aol.com.

DOI:10.1523/JNEUROSCI.5066-07.2008

Copyright $\odot 2008$ Society for Neuroscience $\quad$ 0270-6474/08/281537-09\$15.00/0 mice expressing mutant human APP and TAU genes have recently been developed that display amyloid plaques and tau aggregates (Oddo et al., 2003). We recently reported that Tg2576 APP transgenic mice crossed to a nitric oxide synthase 2 (NOS2) knock-out $\left(\mathrm{NOS}^{-l-}\right.$ ) develop neurofibrillary tangle-like pathology from endogenous mouse tau and show evidence of neurodegeneration assessed by staining for fluoro-jade $\mathrm{C}$ and activated caspase 3 (Colton et al., 2006).

In $\mathrm{AD}$, there are specific neuronal subsets that appear more susceptible to the disease and are affected more severely than others. These include the cholinergic neurons and the neuropeptide Y (NPY) neurons. NPY is a 36 amino acid peptide that acts as a neurotransmitter and has been localized to the CNS and in peripheral nervous tissue (Dumont et al., 1992). NPY expression is particularly high in the interneurons of the dentate gyrus and cornu ammonis 3 (CA3) (Kohler et al., 1986) as well as the mossy fiber pathway linking these two regions. Importantly, significant reductions in NPY neurons in the hippocampal regions of AD brains have been demonstrated (Kowall and Beal, 1988). APP transgenic mice have failed to demonstrate significant loss of NPY neurons; in fact, increased NPY immunoreactivity has been observed in both the V717F APP transgenic (PDAPP) mice (Diez et al., 2000) and the APP23 mice (Diez et al., 2003).

The NOS2 gene encodes inducible NOS (iNOS), one of three isoforms of NOS that generate nitric oxide (NO), and is primarily associated with the innate immune response in all tissues (Kro- 
ncke et al., 2000). Although NO is commonly considered a "cytotoxic" molecule, which is its function during acute disease, $\mathrm{NO}$ is also progrowth and anti-apoptotic (Kroncke et al., 2001; Thomas et al., 2004; Bayir et al., 2005; Brune, 2005; Hewett et al., 2005). This apparent dichotomy of the functions of NO relates to an integration of its tissue concentration, its levels of production, and the wide variety of targets for $\mathrm{NO}$ and its metabolites (Wink et al., 2001). Here, we show that the APPSwDI transgenic mouse crossed to a NOS2 ${ }^{-1-}$ mouse develops further cognitive decline in behavioral tests despite unaltered levels of $A \beta$, has significant neuron loss in disease-relevant regions, and a particularly dramatic loss of NPY neurons, in association with significant tau pathology.

\section{Materials and Methods}

Mouse strains

A bigenic mouse was produced by crossing APPSwDI (Swedish K760N/ M671L, Dutch E693Q, and Iowa D694N) transgenic mice with $\mathrm{NOS}^{-1-}$ (B6 129P2NOS2 $2^{\text {taul Lau/J) }}$ mice (The Jackson Laboratory, Bar Harbor, ME). The phenotype of these individual mice has been reported previously (Laubach et al., 1995; Davis et al., 2004). All mice were genotyped by using standard PCR methods. APPSwDI transgenic mice were also bred to generate age-matched controls. For stereological counts, we also used age-matched wild-type and $\mathrm{NOS}_{2}{ }^{-1-}$ mice.

\section{Behavioral analyses}

APPSwDI $(n=9)$ and APPSwDI/NOS2 ${ }^{-1-}(n=29)$ mice were tested at 52-56 weeks using both the $2 \mathrm{~d}$ radial-arm water maze (Alamed et al., 2006) and the Barnes maze (Xu et al., 2007). The $2 \mathrm{~d}$ radial-arm water maze has been described in detail previously (Alamed et al., 2006). Briefly, a six arm maze is submerged in a pool of water, and a platform is placed at the end of one arm. The mouse receives 15 trials per day for $2 \mathrm{~d}$ and on each trial is started in a different arm while the arm containing the platform remains the same for each mouse. Using visual cues around the room, the mouse learns the position of the escape platform. The first 10 trials are considered training and alternate between a visible and a hidden platform. The final trials for day 1 and all trials on day 2 use a hidden platform. The number of errors (incorrect arm entries) are counted over a $1 \mathrm{~min}$ period. The errors are averaged over three trials resulting in 10 blocks for the $2 \mathrm{~d}$ period.

The Barnes maze has also been described previously (Barnes, 1979; Xu et al., 2007). Briefly, this task was performed over a $5 \mathrm{~d}$ period and used a circular table with eight equidistant holes around the perimeter. An escape box was placed under an escape hole, the location of which remained constant over the $5 \mathrm{~d}$ for each mouse. Mice were tested twice daily for $5 \mathrm{~d}$. The number of nose pokes into incorrect holes was counted on each trial. The two trials on each day were averaged to provide one value per day. All behavioral tests were performed with genotypes blinded to the investigator.

\section{Tissue processing and histological methods}

Mice were injected with a lethal dose of ketamine and perfused intracardially with $25 \mathrm{ml}$ of normal saline. Brains were rapidly removed and bisected in the midsagittal plane. One half was immersion fixed in either $70 \%$ ethanol (APPSwDI, $n=4$; APPSwDI/NOS2 ${ }^{-1-}, n=4$ ) or $4 \%$ paraformaldehyde (APPSwDI, $n=4$; APPSwDI/NOS2 ${ }^{-1-}, n=8$ ). One half was snap frozen in liquid nitrogen and stored at $-80^{\circ} \mathrm{C}$. The hemibrains fixed in $70 \%$ ethanol were embedded in paraffin, and $8 \mu \mathrm{m} \mathrm{sec}-$ tions were cut using a microtome. The hemibrains fixed in $4 \%$ paraformaldehyde were incubated for $24 \mathrm{~h}$ in 10,20 , and $30 \%$ sucrose sequentially to cryoprotect them. Frozen sagittal sections $(25 \mu \mathrm{m})$ were then collected using a sliding microtome and were stored at $4^{\circ} \mathrm{C}$ in PBS with sodium azide to prevent microbial growth. Eight equally spaced sections $\sim 600 \mu \mathrm{m}$ apart were selected for free-floating immunohistochemistry for neuronal-specific nuclear protein (NeuN) (mouse monoclonal, 1:3000; Chemicon, Temecula, CA), NPY (rabbit polyclonal, 1:30,000; Chemicon), paired helical filament (PHF)-tau (AT8, a mouse monoclonal for PHF-tau recognizing phosphorylated Ser202 in tau,
1:150; Pierce Endogen, Rockford, IL), total tau (tau-5, mouse monoclonal anti-tau 5, 1:1000; Calbiochem, La Jolla, CA), cleaved caspase-3 (rabbit polyclonal, 1:300; Cell Signaling Technology, Beverley, MA), or CD45 (rat monoclonal; AbD Serotec, Raleigh, NC). The method for freefloating immunohistochemistry has been described previously (Wilcock et al., 2004a). Also, eight equally spaced sections were mounted on slides and stained with $1 \%$ thioflavine-S, $0.0001 \%$ Fluoro-Jade C in $0.1 \%$ acetic acid or terminal deoxynucleotidyl transferase-mediated biotinylated UTP nick end labeling (TUNEL) [using Dead-End Colorimetric TUNEL System (Promega, Madison, WI) and stained according to manufacturer instructions].

Four equally spaced sections spaced $\sim 1.2 \mathrm{~mm}$ apart were taken from three mice of each genotype and mounted on slides. Double immunofluorescence was performed for neuron-specific $\beta$-tubulin (rabbit polyclonal, 1:1000; Abcam, Cambridge, MA) and AT8. Appropriate Alexa fluor-conjugated secondary antibodies were used.

$\mathrm{A} \beta$ immunohistochemistry was performed on $70 \%$ ethanol fixed 8 $\mu \mathrm{m}$ paraffin-embedded sections. Briefly, sections were deparaffined in xylene and rehydrated. After incubations in hydrogen peroxide and detergent, sections were incubated overnight at $4^{\circ} \mathrm{C}$ with primary antibody (rabbit polyclonal anti-A $\beta \mathrm{N}$ terminal, 1:3000; Biosource, Camarillo, CA). The sections were then incubated with biotinylated anti-rabbit secondary antibody (1:3000; Vector Laboratories, Burlingame, CA) for $2 \mathrm{~h}$ at room temperature followed by incubation in streptavidin $\mathrm{ABC}$ (Vectastain elite ABC kit; Vector Laboratories). The peroxidase was developed using a DAB substrate kit (Vector Laboratories).

\section{Quantification}

Percentage immunoreactive area. Images of the immunohistochemical stain were collected using the $20 \times$ objective lens on the Nikon (Tokyo, Japan) Eclipse TE200 microscope with a Nikon DXM1200 digital camera attached. Images from the frontal cortex, CA1, CA3, dentate gyrus, subiculum, and thalamus were collected from each section. An average of four to five sections for each animal were analyzed. Using the Image-Pro Plus software, we identified the positive stain on two to three representative images, and the red, green, and blue values were saved to a file. This file was then applied to all images to yield measurements of percentage area occupied with positive stain and average intensity of the positive stain for every image. Anatomical landmarks on the sections were used to ensure that the same region was assessed for each section examined. Images for each marker were also collected on the same day to ensure the same illumination on every image. The data were then exported to an Excel spreadsheet (Microsoft, Seattle, WA) where statistical analysis was performed.

Stereological analysis. Neurons that were immunopositive for $\mathrm{NeuN}$ or NPY were counted in the hippocampus, CA3, and the subiculum using the optical fractionator method of stereological counting (West et al., 1991) with commercially available stereological software (StereoInvestigator; MBF Bioscience, Williston, VT). A systematic random sampling of sections throughout the left hippocampus were stained as described above and coded to ensure blinding. The regions of interest were defined using specific landmarks within the hippocampus to maintain consistency. A grid was placed randomly over the region of interest slated for counting. At regularly predetermined positions of the grid, cells were counted within three-dimensional optical dissectors. Within each dissector, a $1 \mu \mathrm{m}$ guard distance from the top and bottom of the section surface was excluded. Section thickness was measured regularly on all collected sections to estimate the mean section thickness for each animal after tissue processing and averaged $12.34 \pm 0.32 \mu \mathrm{m}$ for all sections analyzed. The total number of neurons was calculated using the following equation: $N=Q^{-} \times 1 /$ ssf $\times 1 /$ asf $\times 1 /$ hsf, where $N$ is total neuron number, $Q^{-}$is the number of neurons counted, ssf is section sampling fraction, asf is the area sampling fraction, and hsf is the height sampling fraction.

\section{A $\beta$ ELISA}

Soluble and insoluble pools of $A \beta 1-40$ and $A \beta 1-42$ were measured by sandwich ELISA as described previously (Miao et al., 2005). Briefly, frozen hemibrains were pulverized using a mortar and pestle on dry ice. Samples were homogenized using carbonate for the soluble $\mathrm{A} \beta$ pool. 
A

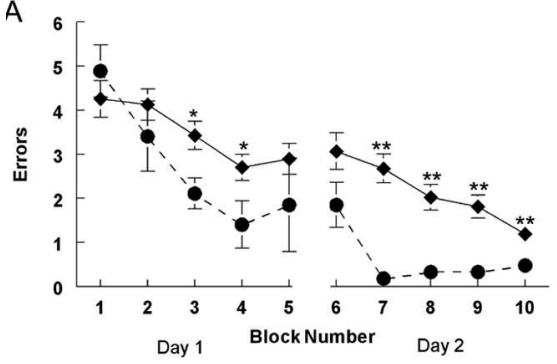

C

\begin{tabular}{|c|c|c|c|c|c|c|}
\hline $\mid$\begin{tabular}{l|l} 
Genotype \\
\end{tabular} & 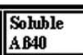 & \begin{tabular}{|l} 
Solohble \\
AB42
\end{tabular} & 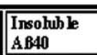 & 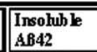 & 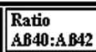 & $\mid \begin{array}{l}\text { Ratio insoluble: } \\
\text { soluble }\end{array}$ \\
\hline$\overline{\overline{\text { APPSSWDI }}}$ & $\mid 1229+135$ & 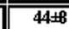 & 243774488 & $11662+224$ & $\overline{\mid \overline{11 \pm 1}}$ & $21+3$ \\
\hline $\mid$ & \begin{tabular}{|l|l}
$1160 \pm 59$ \\
\end{tabular} & $\overline{411 \pm 3}$ & $25882 \pm \pm 424$ & $\overline{4451}$ & $\overline{17 \pm 2}$ & $\overline{3 \pm 4}$ \\
\hline
\end{tabular}

Figure 1. APPSWDI/NOS2 ${ }^{-1-}$ mice have significant spatial memory impairment compared with the APPSwDI mice with no significant difference in $A \beta$ levels. $A$, Two day radial-arm water maze task. Mice receive 15 trials per day, and each block represents the average of three trials. The circles and dashed line indicate data from the APPSwDI mice $(n=9)$, and the diamonds and solid line indicate data from the APPSwDI/NOS2 $2^{-1-}$ mice $(n=29) .{ }^{*} p<0.05$, for the individual data points; ${ }^{* *} p<0.01$, for the individual data points. $\boldsymbol{B}$, Barnes maze. Mice receive two trials per day for $5 \mathrm{~d}$, and the two trials are averaged to give a single value for each day. The circles and dashed line indicate data from the APPSwDI mice $(n=9)$, and the diamonds and solid line indicate data from the APPSwDI/NOS2 ${ }^{-1-}$ mice $(n=29) .{ }^{* *} p<0.01$, for the individual data points. $C, A \beta$ ELISA from brain lysates \pm SEs. No significant differences were found for total $A \beta$ or soluble and insoluble $A \beta 40$ and $A \beta 42$ between APPSWDI/NOS2 $2^{-1-}$ and APPSWDI mice.

This sample was centrifuged at high speed and the supernatant removed. The remaining pellet was then homogenized with guanidine for the insoluble $A \beta$ pool. Again, this sample was centrifuged at high speed and the supernatant extracted. Total A $\beta 1-40$ and 1-42 levels were obtained by adding the values of the soluble and insoluble levels.

\section{Western blotting}

Protein was extracted from pulverized brain powder and quantified using the BCA protein assay kit (Pierce) and performed according to manufacturer instructions. Protein samples $(10 \mu \mathrm{g})$ from each brain lysate were run on a denaturing $4-20 \%$ SDS-PAGE gel. The gel was then transferred onto a nitrocellulose membrane. The transferred membrane was then blocked in $5 \%$ nonfat milk and incubated overnight at $4^{\circ} \mathrm{C}$ in AT8 anti-PHF tau antibody (1:150) diluted in 5\% nonfat milk. The following day, the membrane was washed in TBST (Tris-buffered saline with $1 \%$ Tween 20) and incubated for $1 \mathrm{~h}$ in HRP-conjugated anti-mouse secondary antibody (1:2000) dissolved in 5\% nonfat milk. The membrane was then washed in TBST and developed using the ECL advance Western blotting detection kit (GE Healthcare, Buckinghamshire, UK). Autoradiography film was exposed to the blot and developed. The blot was stripped using Restore stripping buffer (Pierce) and was then reprobed using the above protocol for glyceraldehyde-3-phosphate dehydrogenase (GAPDH) (mouse anti-GAPDH, 1:10,000; Advanced ImmunoChemical, Long Beach, CA) to ensure accurate gel loading. Western blot images were captured using the Kodak Image Station 440 (Carestream Health, Rochester, NY), and densitometry analysis was performed using the Kodak Molecular Imaging software (Carestream Health).

\section{Statistics}

The significance of behavioral changes over time was analyzed by oneway ANOVA. Significance of genotype-specific changes was analyzed by the unpaired Student's $t$ test or two-way ANOVA. The slope of the relationship between number of errors and time was measured using regression analysis and compared across genotypes using two-way ANOVA. All immunohistochemical, stereological, ELISA, and Western blot data were analyzed by one-way ANOVA. The GraphPad Prism 4 program (GraphPad, San Diego, CA) was used to perform all statistical analyses.

\section{Results}

APPSwDI and APPSwDI/NOS2 $2^{-/-}$mice were tested for spatial memory at 52-56 weeks of age using two different behavioral assays that have been shown previously to detect spatial learning and memory deficits in APP transgenic mice (Wilcock et al., 2004b). Figure $1 \mathrm{~A}$ shows that the APPSwDI/NOS2 ${ }^{-1-}$ mice make significantly more errors in the radial-arm water maze than the APPSwDI mice (two-way ANOVA; $p<0.005)$. This difference is particularly apparent on day 2 when the APPSwDI mice are making less than one error, indicating acquisition and retrieval of the task, whereas the APPSwDI/ NOS2 ${ }^{-1-}$ mice are still making two errors or more. Regression analysis of the slopes also showed significant differences $(p<$ 0.05 ) suggesting slowed learning and/or retrieval of the task by the APPSwDI/ NOS2 ${ }^{-1-}$ mice. We also performed the Barnes maze, another spatial memory test previously shown to detect deficits in APP transgenic mice (Xu et al., 2007). Figure $1 B$ shows that the APPSwDI/NOS2 ${ }^{-1-}$ mice are also significantly impaired in this task (two-way ANOVA, $p<0.05$; slope difference, $p<0.001$ ). Control APPSwDI transgenic mice at this age have been shown to have impairment in the Barnes maze and, thus, the removal of NOS2 results in further cognitive decline in these mice. This further decline is not caused by increased amyloid production or deposition, because no change in soluble or insoluble $\mathrm{A} \beta 40$ or $\mathrm{A} \beta 42$ levels were observed (Fig. $1 C$ ).

In $\mathrm{AD}$, tau becomes hyperphosphorylated and redistributed where it aggregates to form neurofibrillary tangles in the neuronal soma. We examined APPSwDI and APPSwDI/NOS2 $2^{-1-}$ mice for presence of abnormally phosphorylated tau using the AT8 antibody. AT8 is a well-recognized antibody used to detect a pathologically relevant hyperphosphorylated tau epitope that is common to both mouse and human tau (Iqbal and GrundkeIqbal, 1997). Protein extracted from the right hemisphere of $\mathrm{NOS}^{-1-}$ (N2), APPSwDI (A) and APPSwDI/NOS2 ${ }^{-1-}$ (AN2) mice were examined by Western blot. Included in the Western blot was protein from P301L mutant human tau transgenic mice (JNPL3) that carry mutant human tau and are known to have extensive tau pathology (Fig. 2 A). Densitometric analysis of the bands, normalized to GAPDH as a loading control, showed increased density in the APPSwDI/NOS2 ${ }^{-1-}$ mice when compared with the parental NOS $2^{-1-}$ and APPSwDI mice (Fig. $2 A$ ). The levels of AT8 were not as high, however, as in the JNPL3 brains. It is important to note that the APPSwDI/NOS2 ${ }^{-1-}$ mice do not have mutant human tau and all tau hyperphosphorylation is that of normal mouse tau. Immunohistochemistry was also performed using the AT8 antibody and analysis of percentage area occupied with positive stain showed significant increases in staining in all regions examined (Fig. $2 B$ ). To ensure that the AT8 antibody labeled intraneuronal protein, we performed double immunocytochemistry for AT8 (Fig. 2C) and neuron-specific $\beta$-tubulin (Fig. 2 D). Merging the two images revealed AT8 staining in the soma of neurons (Fig. 2E).

$\mathrm{A} \beta$ immunohistochemistry showed dense hippocampal staining in APPSwDI (Fig. $3 A$ ) and APPSwDI/NOS $2^{-1-}$ (Fig. $3 C$ ) brains, although no significant differences in regional staining patterns were observed between genotypes. In both APPSwDI and APPSwDI/NOS2 ${ }^{-/-}$mice, the subiculum and thalamus ex- 
hibited particularly dense $\mathrm{A} \beta$ immunoreactivity, part of which was associated with cerebral microvessels within that region. Typical views of the vascular $A \beta$ staining are shown for the thalamus (Fig. 3E, APPSwDI, G, APPSwDI/NOS2 ${ }^{-1-}$ ) and subiculum (Fig. 3I, APPSwDI, $K$, APPSwDI/NOS2 ${ }^{-1-}$ ). We also examined the thalamus and subiculum for tau pathology, particularly in those regions with dense A $\beta$ immunoreactivity. AT8 immunocytochemistry showed only background staining in the APPSwDI mouse (Fig. 3B), whereas we found intense AT8 staining in the hippocampus of the APPSwDI/NOS2 ${ }^{-1-}$ mouse (Fig. 3D). Importantly, the high-density areas of AT8 staining matched the dense regions of $\mathrm{A} \beta$ staining (Fig. 3, compare $C$ and $D$ ). Additional examination at a higher magnification showed processes filled with AT8positive tau associated with blood vessels in the thalamus (Fig. $3 H$ ) and subiculum (Fig. $3 L$ ). These data indicate that hyperphosphorylation of tau occurs in the same location as dense $\mathrm{A} \beta$ deposition but only in APPSwDI mice that also lack the NOS2 gene.

To further distinguish patterns of tau staining, we used the tau 5 antibody that detects total tau levels in mice. Staining for total tau showed a light, diffuse staining pattern in the APPSwDI mouse (Fig. $4 A$ ), whereas in the APPSwDI/NOS2 ${ }^{-1-}$ mouse (Fig. 4D), dense somatodendritic staining was also observed. Somatodendritic redistribution of tau was found in the cortex only in the APPSwDI/NOS2 ${ }^{-/-}$brain and was localized to the same regions where AT8-positive neuronal soma were also observed (Fig. $4 B, E$ ). To determine whether the hyperphosphorylated tau was also aggregated, sections from the APPSwDI and APPSwDI/ $\mathrm{NOS} 2^{-1-}$ mice brains were stained using thioflavin-S, a fluorescent dye that reacts with proteins in the $\beta$-pleated sheet formation. Such proteins would include dense core amyloid deposits and neurofibrillary tangles in AD. Staining of the control APPSwDI mouse showed only staining of blood vessels (Fig. 4C), which is the primary location of amyloid in this mouse. In the APPSwDI/NOS2 ${ }^{-1-}$ mouse, we saw neuronal staining with thioflavin-S (Fig. $4 F$ ), indicating intraneuronal aggregates of protein. Because intraneuronal $\mathrm{A} \beta$ staining was not observed in either mouse strain, it is unlikely that thioflavin-positive staining represented $A \beta$. Instead, the combination of AT8-positive tau and thioflavin-S staining in cortical neuronal soma strongly suggests that tau is both hyperphosphorylated and aggregated in the APPSwDI/NOS2 ${ }^{-1-}$ mouse brain.

The consensus in the field is that tau pathology most closely correlates with disease severity (Arriagada et al., 1992); therefore, we examined the brains in APPSwDI/NOS2 ${ }^{-1-}$ mice for evidence of neurodegeneration. Fluoro-jade $\mathrm{C}$ stains degenerating neurons regardless of the cause of damage (Schmued et al., 2005). We observed numerous fluoro-jade $\mathrm{C}$-positive neurons in the APPSwDI/NOS $2^{-1-}$ mice (Fig. $4 J$ ) compared with only background staining in the APPSwDI mice (Fig. 4G). We then examined two markers of apoptosis to determine whether the neuronal death was attributable to, at least in part, apoptotic mechanisms. Caspase 3 cleavage is a critical step in the apoptotic pathway and has been associated with $\mathrm{A} \beta$-mediated neuronal death ( $\mathrm{Su}$ et al.,
2001; LeBlanc, 2003; Rissman et al., 2004). Immunocytochemistry for cleaved caspase 3 showed positive staining of the neurons in the APPSwDI/NOS2 $2^{-1-}$ mice (Fig. $4 K$ ). TUNEL is a staining technique used to detect fragmented nuclear DNA, which is indicative of apoptosis. We also detected numerous TUNELpositive neurons in the APPSwDI/NOS $2^{-1-}$ mice (Fig. $4 L$ ). Together, all of these data indicate that the APPSwDI/NOS2 ${ }^{-1-}$ has significant neurodegeneration that may involve apoptotic mechanisms.

We then examined neuronal integrity in hippocampal regions. Immunostaining for the neuronal marker, NeuN, on equally spaced sagittal sections revealed thinning of the CA3 (Fig. $5 B, D$ ) and subiculum (Fig. $5 B, F$ ) in APPSwDI/NOS2 ${ }^{-1-}$ mice when compared with the APPSwDI mice (Fig. $5 A, C, E$ ). Stereological counting of neurons was performed using the optical fractionator method in the entire hippocampus as well as in the CA3 region and in the neighboring subiculum (Fig. $2 G$ ). We found a $30 \%$ loss of neurons in the hippocampus $(p<0.05$ compared with wild type, NOS2 ${ }^{-1-}$, or APPSwDI), a 35\% loss of neurons in the subiculum $\left(p<0.01\right.$ compared with wild type, NOS2 ${ }^{-1-}$, or APPSwDI), and a $40 \%$ loss of neurons in the CA3 region of the hippocampus ( $p<0.01$ compared with wild type, NOS2 ${ }^{-1-}$, or APPSwDI).

In $\mathrm{AD}$, it is known that certain subpopulations of neurons are particularly vulnerable to the disease process. We examined NPY neurons as one of these subpopulations. Because of the large microvascular involvement in the pathology of APPSwDI and APPSwDI/NOS2 ${ }^{-1-}$ mice brains, we elected to examine NPY neurons, because these are known to be intimately involved in maintaining cerebrovascular tone at the neurovascular unit (Edvinsson et al., 1987). In the wild-type, NOS2 ${ }^{-1-}$, and the APPSwDI mice, we found strong NPY immunoreactivity of the mossy fiber tract from the dentate gyrus to the CA3 region of the hippocampus along with strong NPY immunoreactivity in the CA1 region (APPSwDI shown in Fig. 6A). A similar pattern of NPY staining was observed in each of the control mice. However, when we examined the APPSwDI/NOS $2^{-1-}$ mice, we found re- 


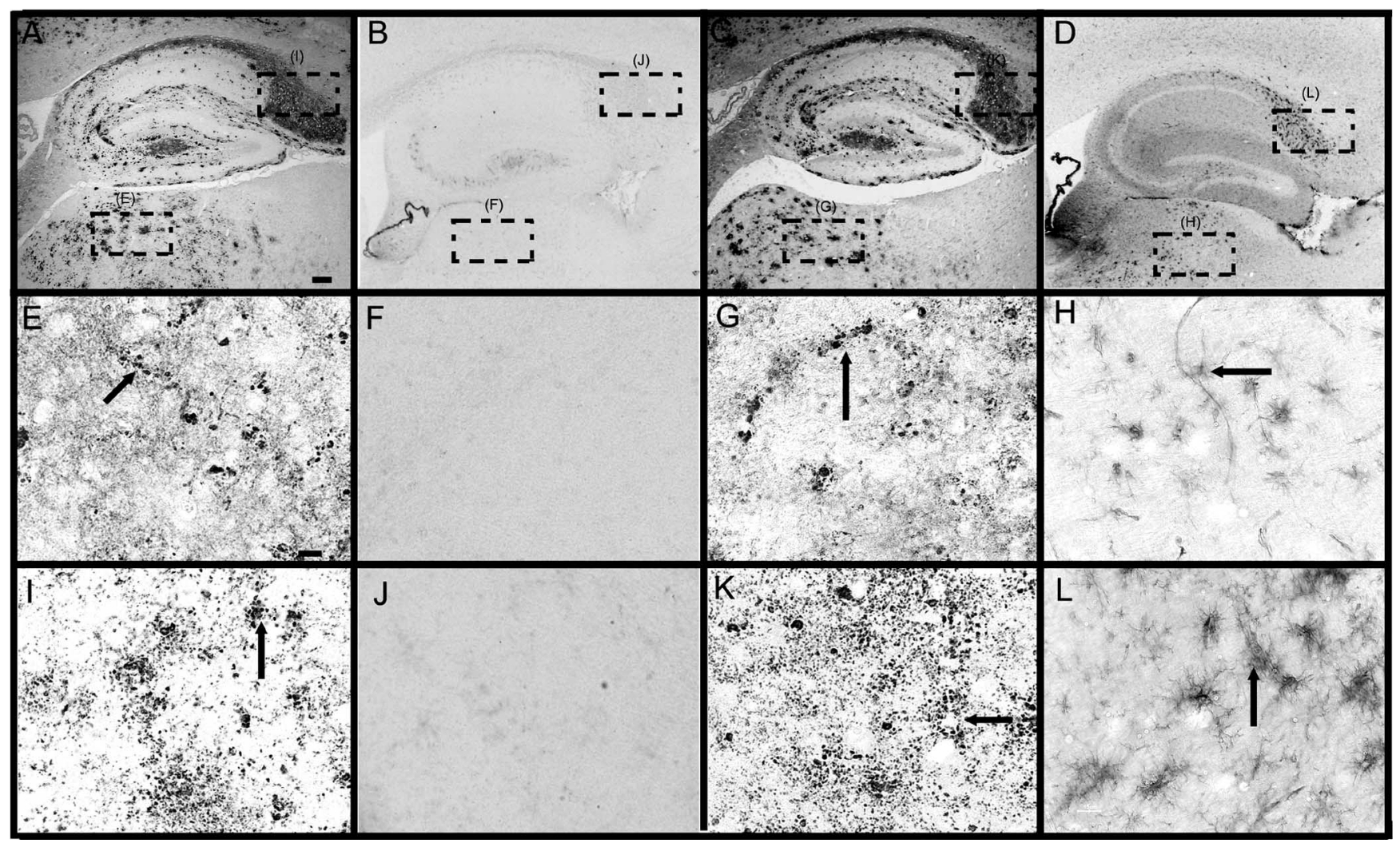

Figure 3. AT8-positive staining is associated with $A \beta$ in blood vessels of the thalamus and subiculum in the APPSwDI/NOS2 $2^{-I-}$ mice. $A, E$, and $I$ show $A \beta$ immunohistochemistry of the APPSwDI mouse, whereas $\boldsymbol{C}, \boldsymbol{G}$, and $\boldsymbol{K}$ show the APPSwDI/NOS2 ${ }^{-\prime-}$ mouse. $\boldsymbol{B}, \boldsymbol{F}$, and $\boldsymbol{J}$ show AT8 immunocytochemistry in the APPSwDI mouse, whereas $\boldsymbol{D}, \boldsymbol{H}$, and $\boldsymbol{L}$ show the APPSwDI/NOS2 mouse. $\boldsymbol{E}$ and $\boldsymbol{I}, \boldsymbol{F}$ and $\boldsymbol{J}, \boldsymbol{G}$ and $\boldsymbol{K}$, and $\boldsymbol{H}$ and $\boldsymbol{L}$ are high-magnification images of the thalamus and subiculum as indicated in $\boldsymbol{A}-\boldsymbol{D}$. The arrows in $\boldsymbol{E}, \boldsymbol{I}, \boldsymbol{G}$, and $\boldsymbol{K}$ indicate examples of perivascular $A \beta$ staining. The arrows in $\boldsymbol{H}$ and $\boldsymbol{L}$ indicate AT8 staining around blood vessels. $\boldsymbol{A}-\boldsymbol{D}$ are $40 \times$ magnification, and $\boldsymbol{E}-\boldsymbol{L}$ are $200 \times$ magnification. Scale bars: (in $\boldsymbol{A}) \boldsymbol{A}-\boldsymbol{D}, 120 \mu \mathrm{m}$; (in $\boldsymbol{E}$ ) $\boldsymbol{E}-\boldsymbol{L}, 25 \mu \mathrm{m}$.

duced intensity of NPY staining throughout the hippocampus (Fig. 6B). Closer examination revealed loss of NPYimmunopositive neurons particularly in the CA3 region (Fig. $6 D$ ) and in the subiculum (Fig. 6F). Stereological counts of NPY neurons revealed a $50 \%$ reduction overall throughout the hippocampus, a $65 \%$ reduction in the $\mathrm{CA} 3$ region, and a $50 \%$ reduction in the subiculum (Fig. $6 G$ ) in the APPSwDI/NOS2 ${ }^{-1-}$ mice compared with wild-type mice brain (in all cases, $p<0.001$, when compared with wild-type, NOS2 ${ }^{-1-}$, or APPSwDI mice). Interestingly, we observed a statistically significant $20 \%$ reduction in NPY neurons in the subiculum of the APPSwDI, although the level of neuronal loss remained significantly greater than the NPY neuronal loss in the APPSwDI/NOS2 ${ }^{-1-}$ mice. This is likely because of the extremely high levels of microvascular amyloid deposition in subiculum.

\section{Discussion}

Replication of AD-like pathology in mice by the sole accumulation of amyloid peptides leading to abnormal tau pathology, neuronal loss, and cognitive dysfunction has proven to be elusive. Incomplete pathological models that show abundant amyloid deposition with little or sparse tau pathology, rare neuronal loss, and limited changes in learning and memory behaviors have been most commonly generated (Games et al., 1995; Hsiao et al., 1996; Hsiao, 1998; Holcomb et al., 1998). We report here a method to progress an amyloid depositing APP transgenic mouse to more complete Alzheimer's-like pathology with robust behavioral deficits. This is accomplished by genetically removing NOS2, and its iNOS protein product in mice that concomitantly express mutated human APP and deposit A $\beta$. These mice appear to closely replicate the human disease showing native tau pathology and neuron loss in the presence of amyloid deposition. Particularly, one subset of neurons known to be vulnerable in human $\mathrm{AD}$ is also particularly vulnerable in this mouse model, the NPY neurons.

The role of iNOS in neuropathology has been associated with neuronal damage and death. Higher levels of NO (>400 nM), as observed in eradication of pathogens during an acute immune response, have pathophysiological consequences. At pathogenic levels, p53 activation and the induction of apoptosis can occur (Brown and Bal-Price, 2003; Thomas et al., 2004). Clearly, the exposure of cells to high levels of NO in an acute manner will produce cell damage and death (Dawson and Dawson, 1998). However, using cyclic GMP activity, a well described surrogate marker of the action of NO, Duport and Garthwaite (2005) have challenged the idea that iNOS production by immune cells in brain slice culture kills adjacent cells. Numerous studies have also shown that iNOS activity can be neuroprotective ( $\operatorname{Sinz}$ et al., 1999; Kroncke et al., 2000; Ciani et al., 2002; Tang et al., 2006). For example, Sinz et al. (1999) reported that cognitive behavioral responses after head injury are worse in iNOS knock-out mice than in wild-type controls. The large, long-term functional loss in these mice is accompanied by decreased brain ascorbate levels leading to brain oxidative stress (Bayir et al., 2005). A fourfold decrease in hippocampal levels of iNOS is observed in aged spontaneously hypertensive rats that also demonstrate significantly poorer performance in the Morris water maze compared with age-matched control rats (Huang et al., 2006). Again, the decreased iNOS levels were associated with increased oxidative 
stress. Our present data on the APPSwDI/ NOS $2^{-1-}$ mouse and our previously published study on the Tg2576 APP mouse on a NOS2 knock-out background (Colton et al., 2006) add to the increasing evidence suggesting that NO generated by iNOS under conditions of long-term injury or disease reduces functional loss and mitigates pathological changes in brain (Akassoglou, 2005; Culmsee et al., 2005; Pannu and Singh, 2006).

We have shown previously that NOS2 removal in the APPSw (Tg2576) mouse results in tau hyperphosphorylation and evidence of neurodegeneration (Colton et al., 2006). This original report focused primarily on the critical characterization of the tau pathology, because this was one of the first reports on initiation of normal mouse tau pathology in an APP transgenic mouse. In the current study, we have not only replicated these findings in another APP transgenic mouse, but we also have now shown that genetic removal of iNOS results in a further cognitive impairment, loss of neurons, and, more specifically, a dramatic loss of the NPY neurons. Also of significance is that the current report uses an APP transgenic mouse, which has high microvascular amyloid deposition, whereas our previous report used an APP transgenic mouse model, which has primarily parenchymal amyloid deposition. In both cases, the highest density of tau pathology was in association with the amyloid.

In human $\mathrm{AD}$ brains, tau hyperphosphorylation and aggregation has been linked to neuronal damage and loss and is predicted to be a downstream effect of $A \beta$ action (Iqbal et al., 1994; Braak and Braak, 1998; Binder et al., 2005). It is also impor-

tant to note than in $\mathrm{AD}$, it is normal human tau that becomes pathological and, as yet, no mutations have been located in the tau gene in $\mathrm{AD}$. This is in contrast to frontotemporal dementia, where numerous mutations in tau, producing the same tau pathology as AD in the absence of amyloid, have been found (Goedert, 2005). Although transgenic mouse models expressing mutated human tau with amyloid have been useful to study some interactions between amyloid and tau (Lewis et al., 2001; Oddo et al., 2003), they are not ideal, because the tau pathology generated by mutated human tau can occur independently of amyloid (Lewis et al., 2000). In the APPSwDI/NOS2 ${ }^{-1-}$ mouse, we found hyperphosphorylation and aggregation of the native mouse tau in regions where amyloid deposition is particularly dense. In this bigenic mouse, the most dense regions of amyloid deposition are the small blood vessels of the thalamus and subiculum (Fig. 3) (Davis et al., 2004). The tau pathology appears in processes within close proximity of these blood vessels that contribute to the neurovascular unit. In human AD where capillary amyloid angiopathy is particularly prevalent, it has been shown that tau also accumulates in these areas (Oshima et al., 2006).

An important aspect of human $\mathrm{AD}$ that results from the other pathologies of the disease is hippocampal and cortical neuron loss. This is an outcome that, for the most part, has eluded scientists working on $\mathrm{AD}$ transgenic mouse models. In fact, only one APP transgenic mouse has been shown to have significantly decreased neuron number (Calhoun et al., 1998). In the mouse model that has provided the most information to date regarding amyloid and tau interactions, the triple transgenic mouse model (Oddo et al., 2003), no neuron loss is observed (Morrissette and LaFerla, 2007). We see significant neuron loss in the APPSwDI/ NOS2 $2^{-1-}$ mice as quantified by stereological neuron counting in the hippocampus, the CA3 region, and the subiculum. Although it is unclear why removal of the NOS2 gene in the APPSwDI mouse results in progression from amyloid pathology to amyloid and tau pathology and neuronal loss, a possible reason is the differences between mouse and human iNOS expression and nitric oxide production. It has been known for some time that human macrophages in vitro produce significantly less $\mathrm{NO}$ when stimulated under conditions that produce high NO levels in mouse macrophages in vitro (Weinberg et al., 1995; Colton et al., 1996). Also, more recently, it has been shown that microglia isolated from mice expressing the human NOS2 gene have signifi- 

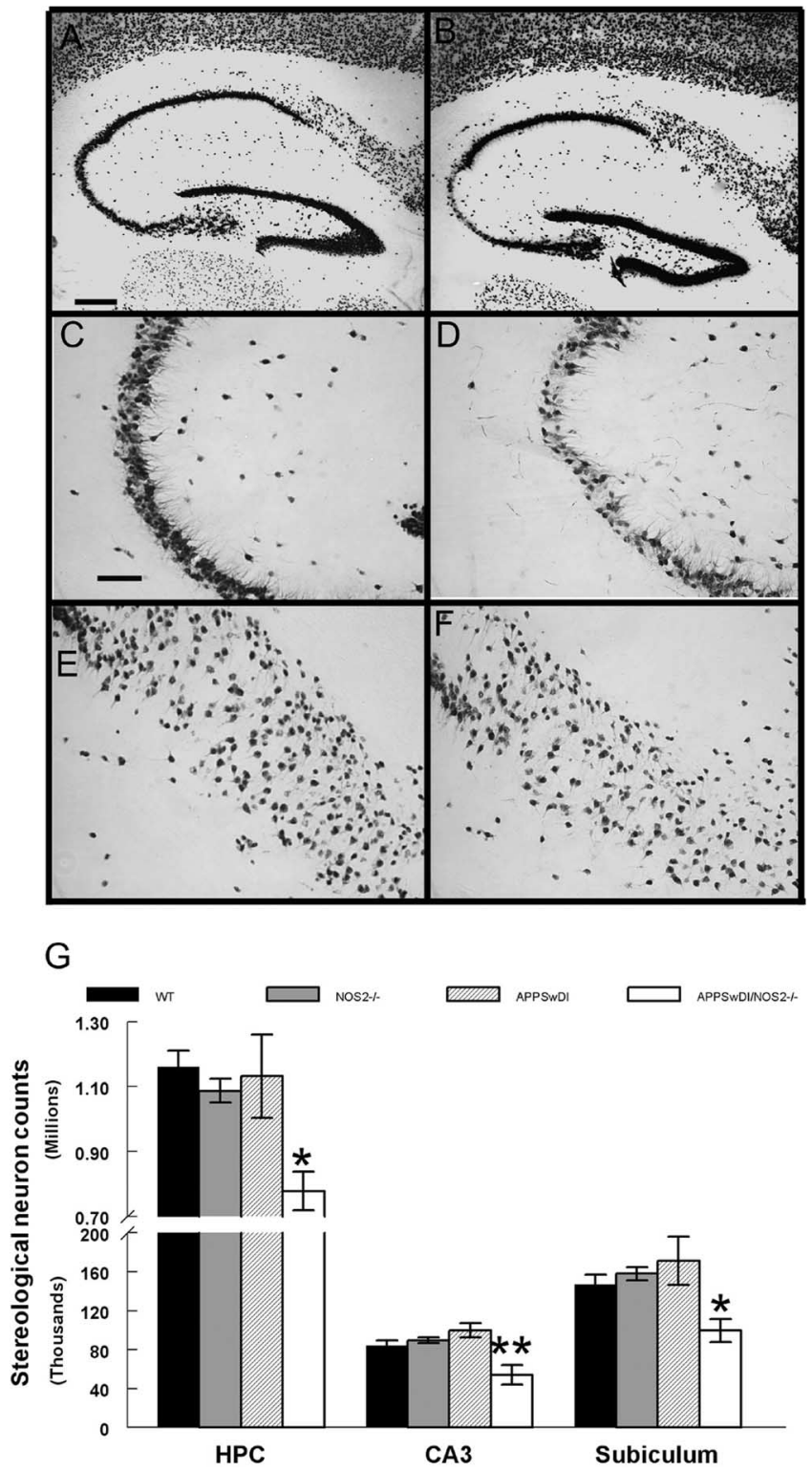

Figure 5. Significant neuron loss is observed in the APPSWDI/NOS2 ${ }^{-1-}$ mouse. $A-F$, NeuN immunocytochemistry in the APPSWDI $(\boldsymbol{A}, \boldsymbol{C}, \boldsymbol{E})$ and the APPSWDI/NOS2 ${ }^{-1-}$ mouse $(\boldsymbol{B}, \boldsymbol{D}, \boldsymbol{F}) \cdot \boldsymbol{A}$, $\boldsymbol{B}$, Whole hippocampus ( $40 \times$ magnification). Scale bar, $120 \mu \mathrm{m}$. C, D, CA3 region. $\boldsymbol{E}, \boldsymbol{F}$, Subiculum (100 $\times$ magnification). Scale bar, $50 \mu \mathrm{m}$. G, Stereological quantification of NeuN staining in the hippocampus, $C A 3$, and subiculum. ${ }^{*} p<0.05$, compared with all other genotypes; ${ }^{* *} p<0.01$, compared with all other genotypes.

cantly lower NO production than wild-type mouse microglia after the same stimulation (Vitek et al., 2006). These data coupled with the multiple differences that have been observed in the promoter region of the human versus the mouse NOS2 gene support the idea that NO production may be significantly different between mouse and human microglia. One hypothesis could be that high levels of NO production in APP transgenic mice are neuroprotective, and therefore pathology does not progress beyond amyloid deposition. By removing iNOS, the nitric oxide balance is now more like the human brain, and therefore amyloid deposition can stimulate the other $\mathrm{AD}$ pathologies. Additional studies will be required to test this hypothesis.

NPY is an important peptide neurotransmitter in interneurons throughout the brain and has been implicated in learning and memory. Injection of NPY into the brains of CD-1 mice
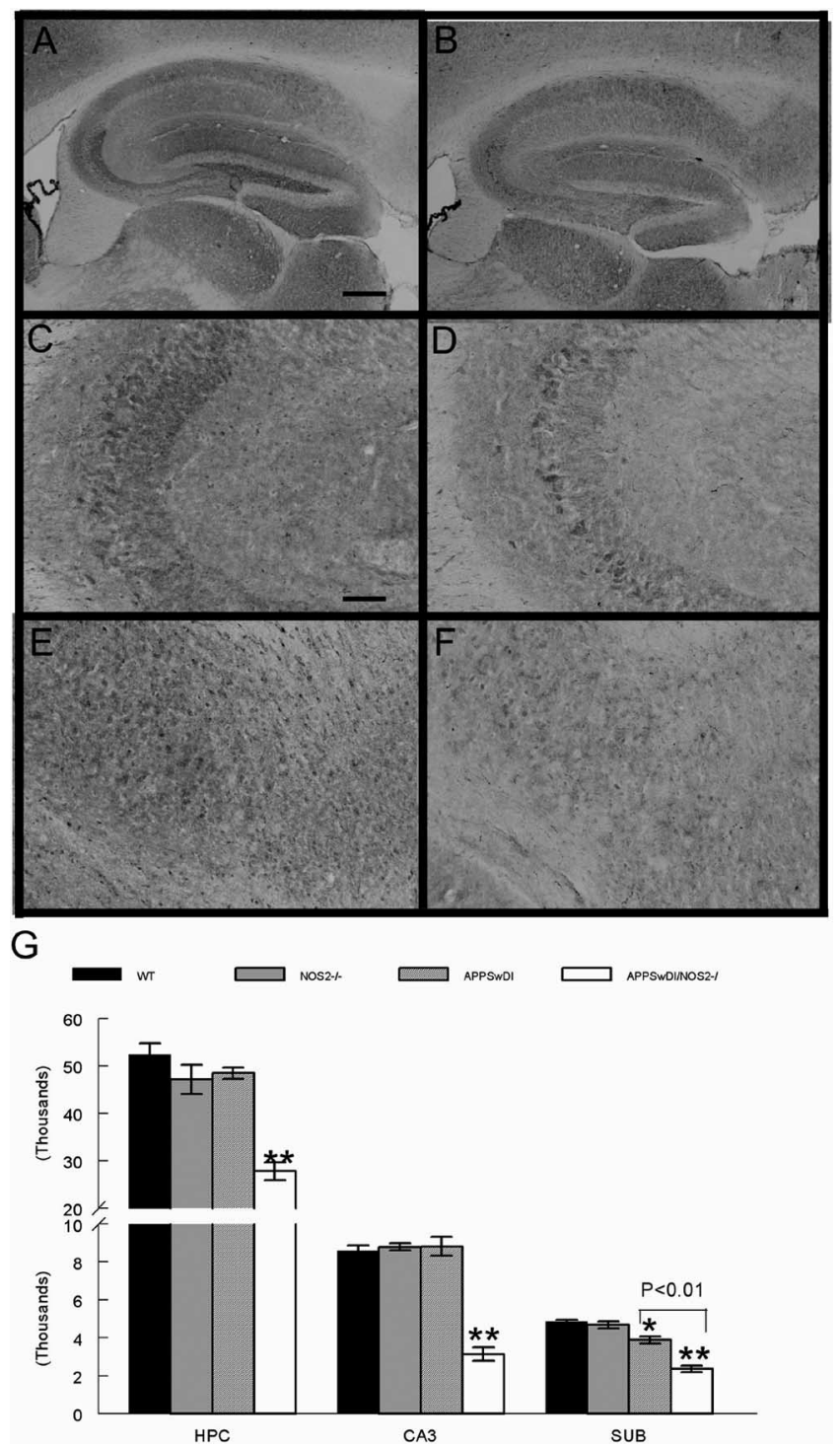

Figure 6. Significant neuropeptide $\mathrm{Y}$ neuron loss is observed in the APPSwDI/NOS2 ${ }^{-1-}$ mouse. $A-F, N P Y$ immunocytochemistry in the APPSwDI $(A, C, E)$ and the APPSwDI/NOS2 ${ }^{-1}$ mouse $(\boldsymbol{B}, \boldsymbol{D}, \boldsymbol{F}) . \boldsymbol{A}, \boldsymbol{B}$, Whole hippocampus (40× magnification). Scale bar, $120 \mu \mathrm{m} . \boldsymbol{C}, \boldsymbol{D}$, CA3 region. $\boldsymbol{E}, \boldsymbol{F}$, Subiculum (200× magnification). Scale bar, $25 \mu \mathrm{m}$. $\boldsymbol{G}$, Stereological quantification of neuronal NPY staining in the hippocampus, CA3, and subiculum. ${ }^{*} p<0.05$ compared with all other genotypes; ${ }^{* *} p<0.01$, compared with all other genotypes.

improves spatial memory (Flood et al., 1987), whereas NPY Y2 receptor knock-out mice (NPY Y2 ${ }^{-l-}$ ) demonstrate impaired cognition (Redrobe et al., 2004). CSF NPY levels (Minthon et al., 1990; Martignoni et al., 1992) and NPY-positive neurons in the hippocampus are significantly reduced in AD brains (Kowall and Beal, 1988). Furthermore, NPY has been shown to affect cerebral blood flow causing either direct vasoconstriction or indirect NOmediated vasodilation (Suzuki et al., 1989) (Kobari et al., 1993). Because the APPSwDI/NOS2 ${ }^{-1-}$ mouse has a large vascular component to its pathology, we examined the NPY neurons in this mouse model in the same brain regions where total neuronal number was reduced. Using stereological methods, we found a more profound loss of NPY neurons than overall losses in total neuron numbers. This would suggest that the NPY neuron population is particularly vulnerable in the APPSwDI/NOS2 $2^{-1-}$ mice as is the case in human AD.

To summarize, we have progressed amyloid pathology in an 
APP transgenic mouse to further develop tau pathology and neuronal loss by genetically deleting the NOS2 gene. This mouse represents a significant advancement over commonly used mouse models of Alzheimer's disease, because the tau pathology is that of normal mouse tau. Furthermore, in addition to increased loss of total neurons, at least one of the well-known vulnerable neuron populations in AD, the NPY neurons, are depleted in this mouse model. The APPSwDI/NOS2 ${ }^{-1-}$ mouse now provides the means to truly test the amyloid hypothesis of $\mathrm{AD}$ and also provides a potentially useful model in which to test therapeutic interventions for the treatment of AD.

\section{References}

Akassoglou K (2005) Nerve growth factor-independent neuronal survival: a role for NO donors. Mol Pharmacol 68:952-955.

Alamed J, Wilcock DM, Diamond DM, Gordon MN, Morgan D (2006) Two-day radial-arm water maze learning and memory task; robust resolution of amyloid-related memory deficits in transgenic mice. Nat Protoc 1:1671-1679.

Arriagada PV, Growdon JH, Hedley-Whyte ET, Hyman BT (1992) Neurofibrillary tangles but not senile plaques parallel duration and severity of Alzheimer's disease. Neurology 42:631-639.

Barnes CA (1979) Memory deficits associated with senescence: a neurophysiological and behavioral study in the rat. J Comp Physiol Psychol 93:74-104.

Bayir H, Kagan VE, Borisenko GG, Tyurina YY, Janesko KL, Vagni VA, Billiar TR, Williams DL, Kochanek PM (2005) Enhanced oxidative stress in iNOS-deficient mice after traumatic brain injury: support for a neuroprotective role of iNOS. J Cereb Blood Flow Metab 25:673-684.

Binder LI, Guillozet-Bongaarts AL, Garcia-Sierra F, Berry RW (2005) Tau, tangles, and Alzheimer's disease. Biochim Biophys Acta 1739:216-223.

Braak H, Braak E (1998) Evolution of neuronal changes in the course of Alzheimer's disease. J Neural Transm Suppl 53:127-140.

Brown GC, Bal-Price A (2003) Inflammatory neurodegeneration mediated by nitric oxide, glutamate, and mitochondria. Mol Neurobiol 27:325-355.

Brune B (2005) The intimate relation between nitric oxide and superoxide in apoptosis and cell survival. Antioxid Redox Signal 7:497-507.

Calhoun ME, Wiederhold KH, Abramowski D, Phinney AL, Probst A, Sturchler-Pierrat C, Staufenbiel M, Sommer B, Jucker M (1998) Neuron loss in APP transgenic mice. Nature 395:755-756.

Ciani E, Guidi S, Bartesaghi R, Contestabile A (2002) Nitric oxide regulates cGMP-dependent cAMP-responsive element binding protein phosphorylation and Bcl-2 expression in cerebellar neurons: implication for a survival role of nitric oxide. J Neurochem 82:1282-1289.

Colton C, Wilt S, Gilbert D, Chernyshev O, Snell J, Dubois-Dalcq M (1996) Species differences in the generation of reactive oxygen species by microglia. Mol Chem Neuropathol 28:15-20.

Colton CA, Vitek MP, Wink DA, Xu Q, Cantillana V, Previti ML, Van Nostrand WE, Weinberg JB, Dawson H (2006) NO synthase 2 (NOS2) deletion promotes multiple pathologies in a mouse model of Alzheimer's disease. Proc Natl Acad Sci USA 103:12867-12872.

Culmsee C, Gerling N, Landshamer S, Rickerts B, Duchstein HJ, Umezawa K, Klumpp S, Krieglstein J (2005) Nitric oxide donors induce neurotrophin-like survival signaling and protect neurons against apoptosis. Mol Pharmacol 68:1006-1017.

Davis J, Xu F, Deane R, Romanov G, Previti ML, Zeigler K, Zlokovic BV, Van Nostrand WE (2004) Early-onset and robust cerebral microvascular accumulation of amyloid beta-protein in transgenic mice expressing low levels of a vasculotropic Dutch/Iowa mutant form of amyloid betaprotein precursor. J Biol Chem 279:20296-20306.

Dawson VL, Dawson TM (1998) Nitric oxide in neurodegeneration. Prog Brain Res 118:215-229.

Diez M, Koistinaho J, Kahn K, Games D, Hokfelt T (2000) Neuropeptides in hippocampus and cortex in transgenic mice overexpressing V717F betaamyloid precursor protein-initial observations. Neuroscience 100:259-286.

Diez M, Danner S, Frey P, Sommer B, Staufenbiel M, Wiederhold KH, Hokfelt T (2003) Neuropeptide alterations in the hippocampal formation and cortex of transgenic mice overexpressing beta-amyloid precursor protein (APP) with the Swedish double mutation (APP23). Neurobiol Dis 14:579-594.

Dumont Y, Martel JC, Fournier A, St-Pierre S, Quirion R (1992) Neuropeptide $\mathrm{Y}$ and neuropeptide $\mathrm{Y}$ receptor subtypes in brain and peripheral tissues. Prog Neurobiol 38:125-167.

Duport S, Garthwaite J (2005) Pathological consequences of inducible nitric oxide synthase expression in hippocampal slice cultures. Neuroscience 135:1155-1166.

Edvinsson L, Copeland JR, Emson PC, McCulloch J, Uddman R (1987) Nerve fibers containing neuropeptide $\mathrm{Y}$ in the cerebrovascular bed: immunocytochemistry, radioimmunoassay, and vasomotor effects. J Cereb Blood Flow Metab 7:45-57.

Eriksen JL, Janus CG (2007) Plaques, tangles, and memory loss in mouse models of neurodegeneration. Behav Genet 37:79-100.

Flood JF, Hernandez EN, Morley JE (1987) Modulation of memory processing by neuropeptide Y. Brain Res 421:280-290.

Games D, Adams D, Alessandrini R, Barbour R, Berthelette P, Blackwell C, Carr T, Clemens J, Donaldson T, Gillespie F (1995) Alzheimer-type neuropathology in transgenic mice overexpressing V717F beta-amyloid precursor protein. Nature 373:523-527.

Goedert M (2005) Tau gene mutations and their effects. Mov Disord 20:S45-S52.

Hardy J, Selkoe DJ (2002) The amyloid hypothesis of Alzheimer's disease: progress and problems on the road to therapeutics. Science 297:353-356.

Hewett SJ, Espey MG, Uliasz TF, Wink DA (2005) Neurotoxicity of nitroxyl: insights into HNO and NO biochemical imbalance. Free Radic Biol Med 39:1478-1488.

Holcomb L, Gordon MN, McGowan E, Yu X, Benkovic S, Jantzen P, Wright K, Saad I, Mueller R, Morgan D, Sanders S, Zehr C, O'Campo K, Hardy J, Prada CM, Eckman C, Younkin S, Hsiao K, Duff K (1998) Accelerated Alzheimer-type phenotype in transgenic mice carrying both mutant amyloid precursor protein and presenilin 1 transgenes. Nat Med 4:97-100.

Hsiao K (1998) Transgenic mice expressing Alzheimer amyloid precursor proteins. Exp Gerontol 33:883-889.

Hsiao K, Chapman P, Nilsen S, Eckman C, Harigaya Y, Younkin S, Yang F, Cole G (1996) Correlative memory deficits, Abeta elevation, and amyloid plaques in transgenic mice. Science 274:99-102.

Huang Y, Wu L, Xu C, Yang B, Wang R (2006) Increased HO-1 expression and decreased iNOS expression in the hippocampus from adult spontaneously hypertensive rats. Cell Biochem Biophys 46:35-42.

Iqbal K, Grundke-Iqbal I (1997) Mechanism of Alzheimer neurofibrillary degeneration and the formation of tangles. Mol Psychiatry 2:178-180.

Iqbal K, Alonso AC, Gong CX, Khatoon S, Singh TJ, Grundke-Iqbal I (1994) Mechanism of neurofibrillary degeneration in Alzheimer's disease. Mol Neurobiol 9:119-123.

Kobari M, Fukuuchi Y, Tomita M, Tanahashi N, Yamawaki T, Takeda H, Matsuoka S (1993) Transient cerebral vasodilatory effect of neuropeptide $\mathrm{Y}$ mediated by nitric oxide. Brain Res Bull 31:443-448.

Kohler C, Eriksson L, Davies S, Chan-Palay V (1986) Neuropeptide Y innervation of the hippocampal region in the rat and monkey brain. J Comp Neurol 244:384-400.

Kowall NW, Beal MF (1988) Cortical somatostatin, neuropeptide Y, and NADPH diaphorase neurons: normal anatomy and alterations in Alzheimer's disease. Ann Neurol 23:105-114.

Kroncke KD, Suschek CV, Kolb-Bachofen V (2000) Implications of inducible nitric oxide synthase expression and enzyme activity. Antioxid Redox Signal 2:585-605.

Kroncke KD, Fehsel K, Suschek C, Kolb-Bachofen V (2001) Inducible nitric oxide synthase-derived nitric oxide in gene regulation, cell death and cell survival. Int Immunopharmacol 1:1407-1420.

Laubach VE, Shesely EG, Smithies O, Sherman PA (1995) Mice lacking inducible nitric oxide synthase are not resistant to lipopolysaccharideinduced death. Proc Natl Acad Sci USA 92:10688-10692.

LeBlanc AC (2003) Natural cellular inhibitors of caspases. Prog Neuropsychopharmacol Biol Psychiatry 27:215-229.

Lewis J, McGowan E, Rockwood J, Melrose H, Nacharaju P, Van Slegtenhorst M, Gwinn-Hardy K, Murphy MP, Baker M, Yu X, Duff K, Hardy J, Corral A, Lin WL, Yen SH, Dickson DW, Davies P, Hutton M (2000) Neurofibrillary tangles, amyotrophy and progressive motor disturbance in mice expressing mutant (P301L) tau protein. Nat Genet 25:402-405.

Lewis J, Dickson DW, Lin WL, Chisholm L, Corral A, Jones G, Yen SH, Sahara N, Skipper L, Yager D, Eckman C, Hardy J, Hutton M, McGowan E 
(2001) Enhanced neurofibrillary degeneration in transgenic mice expressing mutant tau and APP. Science 293:1487-1491.

Martignoni E, Blandini F, Petraglia F, Pacchetti C, Bono G, Nappi G (1992) Cerebrospinal fluid norepinephrine, 3-methoxy-4-hydroxyphenylglycol and neuropeptide Y levels in Parkinson's disease, multiple system atrophy and dementia of the Alzheimer type. J Neural Transm Park Dis Dement Sect 4:191-205.

Miao J, Xu F, Davis J, Otte-Holler I, Verbeek MM, Van Nostrand WE (2005) Cerebral microvascular amyloid beta protein deposition induces vascular degeneration and neuroinflammation in transgenic mice expressing human vasculotropic mutant amyloid beta precursor protein. Am J Pathol 167:505-515.

Minthon L, Edvinsson L, Ekman R, Gustafson L (1990) Neuropeptide levels in Alzheimer's disease and dementia with frontotemporal degeneration. J Neural Transm Suppl 30:57-67.

Morrissette DA, LaFerla FM (2007) Stereological quantification of neuronal cells in the 3XTg-AD mice. Soc Neurosci Abstr 33:888.8.

Nagy Z, Esiri MM, Joachim C, Jobst KA, Morris JH, King EM, Hindley NJ, McDonald B, Litchfield S, Barnetson L, Smith AD (1998) Comparison of pathological diagnostic criteria for Alzheimer disease. Alzheimer Dis Assoc Disord 12:182-189.

Oddo S, Caccamo A, Shepherd JD, Murphy MP, Golde TE, Kayed R, Metherate R, Mattson MP, Akbari Y, Laferla FM (2003) Triple-transgenic model of Alzheimer's disease with plaques and tangles: intracellular Abeta and synaptic dysfunction. Neuron 39:409-421.

Oshima K, Akiyama H, Tsuchiya K, Kondo H, Haga C, Shimomura Y, Iseki E, Uchikado H, Kato M, Niizato K, Arai H (2006) Relative paucity of tau accumulation in the small areas with abundant Abeta42-positive capillary amyloid angiopathy within a given cortical region in the brain of patients with Alzheimer pathology. Acta Neuropathol (Berl) 111:510-518.

Pannu R, Singh I (2006) Pharmacological strategies for the regulation of inducible nitric oxide synthase: neurodegenerative versus neuroprotective mechanisms. Neurochem Int 49:170-182.

Redrobe JP, Dumont Y, Herzog H, Quirion R (2004) Characterization of neuropeptide $\mathrm{Y}, \mathrm{Y}(2)$ receptor knockout mice in two animal models of learning and memory processing. J Mol Neurosci 22:159-166.

Rissman RA, Poon WW, Blurton-Jones M, Oddo S, Torp R, Vitek MP, Laferla FM, Rohn TT, Cotman CW (2004) Caspase-cleavage of tau is an early event in Alzheimer disease tangle pathology. J Clin Invest 114:121-130.

Santacruz K, Lewis J, Spires T, Paulson J, Kotilinek L, Ingelsson M, Guimaraes A, DeTure M, Ramsden M, McGowan E, Forster C, Yue M, Orne J, Janus C, Mariash A, Kuskowski M, Hyman B, Hutton M, Ashe KH (2005) Tau suppression in a neurodegenerative mouse model improves memory function. Science 309:476-481.

Schmued LC, Stowers CC, Scallet AC, Xu L (2005) Fluoro-Jade C results in ultra high resolution and contrast labeling of degenerating neurons. Brain Res 1035:24-31.

Schwab C, Hosokawa M, McGeer PL (2004) Transgenic mice overexpressing amyloid beta protein are an incomplete model of Alzheimer disease. Exp Neurol 188:52-64.
Sinz EH, Kochanek PM, Dixon CE, Clark RS, Carcillo JA, Schiding JK, Chen M, Wisniewski SR, Carlos TM, Williams D, DeKosky ST, Watkins SC, Marion DW, Billiar TR (1999) Inducible nitric oxide synthase is an endogenous neuroprotectant after traumatic brain injury in rats and mice. J Clin Invest 104:647-656.

Su JH, Zhao M, Anderson AJ, Srinivasan A, Cotman CW (2001) Activated caspase-3 expression in Alzheimer's and aged control brain: correlation with Alzheimer pathology. Brain Res 898:350-357.

Suzuki Y, Satoh S, Ikegaki I, Okada T, Shibuya M, Sugita K, Asano T (1989) Effects of neuropeptide $\mathrm{Y}$ and calcitonin gene-related peptide on local cerebral blood flow in rat striatum. J Cereb Blood Flow Metab 9:268-270.

Tang XQ, Yu HM, Zhi JL, Cui Y, Tang EH, Feng JQ, Chen PX (2006) Inducible nitric oxide synthase and cyclooxgenase-2 mediate protection of hydrogen peroxide preconditioning against apoptosis induced by oxidative stress in PC12 cells. Life Sci 79:870-876.

Thomas DD, Espey MG, Ridnour LA, Hofseth LJ, Mancardi D, Harris CC, Wink DA (2004) Hypoxic inducible factor lalpha, extracellular signalregulated kinase, and $\mathrm{p} 53$ are regulated by distinct threshold concentrations of nitric oxide. Proc Natl Acad Sci USA 101:8894-8899.

Vitek MP, Brown C, Xu Q, Dawson H, Mitsuda N, Colton CA (2006) Characterization of $\mathrm{NO}$ and cytokine production in immune-activated microglia and peritoneal macrophages derived from a mouse model expressing the human NOS2 gene on a mouse NOS2 knockout background. Antioxid Redox Signal 8:893-901.

Weinberg JB, Misukonis MA, Shami PJ, Mason SN, Sauls DL, Dittman WA, Wood ER, Smith GK, McDonald B, Bachus KE (1995) Human mononuclear phagocyte inducible nitric oxide synthase (iNOS): analysis of iNOS mRNA, iNOS protein, biopterin, and nitric oxide production by blood monocytes and peritoneal macrophages. Blood 86:1184-1195.

West MJ, Slomianka L, Gundersen HJ (1991) Unbiased stereological estimation of the total number of neurons in thesubdivisions of the rat hippocampus using the optical fractionator. Anat Rec 231:482-497.

Wilcock DM, Rojiani A, Rosenthal A, Levkowitz G, Subbarao S, Alamed J, Wilson D, Wilson N, Freeman MJ, Gordon MN, Morgan D (2004a) Passive amyloid immunotherapy clears amyloid and transiently activates microglia in a transgenic mouse model of amyloid deposition. J Neurosci 24:6144-6151.

Wilcock DM, Rojiani A, Rosenthal A, Subbarao S, Freeman MJ, Gordon MN, Morgan D (2004b) Passive immunotherapy against Abeta in aged APPtransgenic mice reverses cognitive deficits and depletes parenchymal amyloid deposits in spite of increased vascular amyloid and microhemorrhage. J Neuroinflammation 1:24.

Wink DA, Miranda KM, Espey MG, Pluta RM, Hewett SJ, Colton C, Vitek M, Feelisch M, Grisham MB (2001) Mechanisms of the antioxidant effects of nitric oxide. Antioxid Redox Signal 3:203-213.

Xu F, Grande AM, Robinson JK, Previti ML, Vasek M, Davis J, Van Nostrand WE (2007) Early-onset subicular microvascular amyloid and neuroinflammation correlate with behavioral deficits in vasculotropic mutant amyloid beta-protein precursor transgenic mice. Neuroscience 146:98107. 